\title{
Designing a Community-Based Population Health Model
}

\author{
Christopher J. Durovich, MS, MBA ${ }^{1}$ and Peter W. Roberts, MHE, MHA ${ }^{2}$
}

\begin{abstract}
The pace of change from volume-based to value-based payment in health care varies dramatically among markets. Regardless of the ultimate disposition of the Affordable Care Act, employers and public-private payers will continue to increase pressure on health care providers to assume financial risk for populations in the form of shared savings, bundled payments, downside risk, or even capitation. This article outlines a suggested road map and practical considerations for health systems that are building or planning to build population health capabilities to meet the needs of their local markets. The authors review the traditional core capabilities needed to address the medical determinants of health for a population. They also share an innovative approach to community service integration to address the social determinants of health and the engagement of families to improve their own health and well-being. The foundational approach is to connect insurance products, the health care delivery system, and community service agencies around the family's well-being goals using human-centered design strategy.
\end{abstract}

Keywords: population health capabilities, population health infrastructure, clinical integration, community service organization integration, accountable health community, social determinants of health

\section{Introduction}

$\mathbf{T}$ HE PACE OF CHANGE FROM volume-based to value-based payment in health care varies dramatically among markets and within markets. Regardless of the ultimate disposition of the Affordable Care Act, employers and public-private payers will continue, albeit at varying speeds, to increase pressure on providers to take on financial risk in the form of shared savings, bundled payments, downside risk, or even capitation. Additional motivation for hospitals and physicians to transform to population health management comes from the Medicare Access and CHIP Reauthorization Act of 2015, which fundamentally changes Medicare reimbursement for physician services. However, even as health care systems develop capabilities to succeed in value-based models, their current volumebased models will continually face revenue demands to fund operations, capital expansion and replacement, and new initiatives and ventures.

This article outlines a suggested road map and practical considerations for health systems that are building or planning to build population health capabilities. This road map is based on detailed examples from the experience of Children's Health System of Texas (Children's Health) and lessons learned from its own journey.

\section{Context}

Children's Health is an academic pediatric hospital, serving about 200,000 children from north Texas and beyond, which transformed itself into an integrated financing and delivery system beginning in 2012. It led this transformation with: (1) the establishment of a physician-led clinically integrated network called Pediatric Partners for Children that comprises more than 300 private pediatricians and pediatric specialists; (2) the development of licensed insurance vehicles to capture enrolled populations; (3) the building of a comprehensive set of integrated population health management capabilities to support providers of care and the enrolled populations; (4) the creation of a community coalition focused on the health and well-being of children to complement and expand the reach of Children's Health's activities with both enrolled populations and other children in the community; and (5) the formation of an accountable care organization (ACO) with other providers in the market. Children's Health governance and leadership advanced this strategy to improve the organization's performance and likelihood of success as the industry was shifting from volume to value-based care. Children's Health also ensured that the governance structure for the health maintenance organization (HMO), the ACO, and the

\footnotetext{
${ }^{1}$ Office of the CEO and ${ }^{2}$ Population Health and Insurance Services, Children's Health System of Texas, Dallas, Texas.

(c) Christopher J. Durovich and Peter W. Roberts 2018; Published by Mary Ann Liebert, Inc. This article is available under the Creative Commons License CC-BY-NC (http://creativecommons.org/licenses/by-nc/4.0). This license permits non-commercial use, distribution and reproduction in any medium, provided the original work is properly cited. Permission only needs to be obtained for commercial use and can be done via RightsLink.
} 
clinically integrated network included experienced, influential elected physician representatives.

The future state objectives for this integrated system track closely with the Triple Aim Plus:

- Improve quality as measured by pediatric Healthcare Effectiveness Data and Information Set measures and the reduction of variation in practice patterns across physicians in north Texas;

- Improve family health and well-being, with the hypothesis that "enabling families to improve their well-being will lead to improved health for individual children in the context of the family, and ultimately reduce the reactive dependence on acute, episodic medical services" ${ }^{\text {; }}$;

- Improve equity and access to essential health, insurance, and community services for all children in north Texas; and

- Improve medical costs as measured by annual cost trends approaching the consumer price index and outperforming national and state benchmarks.

At this time, all key capabilities have been developed and are operating. The ACO has been operational for 3 years and currently has 78,000 members. The Medicaid HMO became operational in November 2016 with the enrollment of 10,000 children in the STARKids (Supplemental Security Income) program, and the HMO will bid on enrollment of the general Medicaid population when the state of Texas issues its next set of contracts. Children's Health is optimistic that this new and developing set of capabilities will perform well against its future state objectives, given encouraging early results with leading indicators, such as the adoption of treatment protocols, reductions in discretionary use of acute medical services, improvements in self-reported family responses on a well-being quotient, and enrollment in its financing vehicles.

\section{Market Dynamics Guide Both Scope and Scale}

A population health strategy focuses primarily on the local market. Starting the process by identifying and deeply understanding the targeted populations helps an organization determine the scope and scale of capabilities needed, then helps them fine-tune the development of those capabilities.

The authors offer 3 foundational steps to examine the market and assess both opportunities and threats health care systems may face when they assume a role in promoting population health and well-being.

1. Understand the payer dynamics in the local market: What are their capabilities and how are these capabilities currently being deployed? Are any payer organizations interested in creating risk-sharing arrangements? Will the reputation of your organization with local and regional payers facilitate or impede progress in working together?

2. Look at community health needs comprehensively: Too many traditional community health needs assessments have simply been exercises in "checking the box," rather than efforts to deeply understand the specific health and well-being issues of people in the local area. What data exist, and what data may need to be gathered in order to create a comprehensive view of community health needs?

3. Map community assets: What existing organizations, activities, and resources are in place to help popula- tions improve their health and well-being? Where are the gaps, and where is there duplication? What is the competition/collaboration dynamic? How do organizations coalesce around issues of concern to the community?

\section{Capabilities for a Population Health Infrastructure}

Pivoting from a health care delivery system to an organization that manages population health requires capabilities that the organization usually does not currently have. Even if the necessary capabilities exist, the organization must figure out how to deploy them in new ways and integrate the capabilities with the delivery of care. At Children's Health, the development of capabilities included building new functions and repurposing or expanding existing functions. The organization set the goal of building a centralized group of "utility" capabilities that would allow it to serve different populations and product lines, as well as contribute the capabilities in a strategic relationship with affiliated physician organizations.

The capabilities (Fig. 1) Children's Health needed to build a population health infrastructure included core capabilities that were needed from the outset, as well as "adjacent" capabilities that would enhance performance of the infrastructure and could be developed later.

\section{Core Capabilities}

\section{Insurance and/or other risk-bearing vehicles}

Some health systems can negotiate a balanced portfolio of third-party payer opportunities for shared risk without entering the insurance business, providing them more flexibility in how quickly their new capabilities need to be built and launched. A provider-owned, or joint-ventured HMO can provide important opportunities in a value-based payment environment by giving the organization access to the full premium dollar and direct interactions with the purchaser, the provider network, and the enrolled consumers. The drawback is that the strategy requires all capabilities to be developed prospectively and scaled to meet regulatory and market demands. Health systems ordinarily lack "bench strength" in insurance capabilities within their management ranks, requiring significant outside hiring and delay in implementation. Moreover, the HMO enterprise within a provider system is by definition an entrepreneurial activity, and its pace of growth and capital requirements often create a cultural divide within its parent organization.

Children's Health faced a number of challenges in the local market that led it to establish a Medicaid/CHIP HMO — most critical the paucity of local market payers that were willing to entertain risk arrangements. The organization also faced the possibility of being excluded from local networks because of the high cost structure of its inpatient facility, growing local competition, and new competition from adult health systems entering the pediatric market.

Whatever form the financing vehicles take, the key challenge of integrating the risk-bearing vehicle and physician practices to improve performance requires financial incentives cocreated with physicians tied to quality and cost indicators, rewarding both improvement as well as comparisons to benchmarks. The precursor activity is sharing data with 


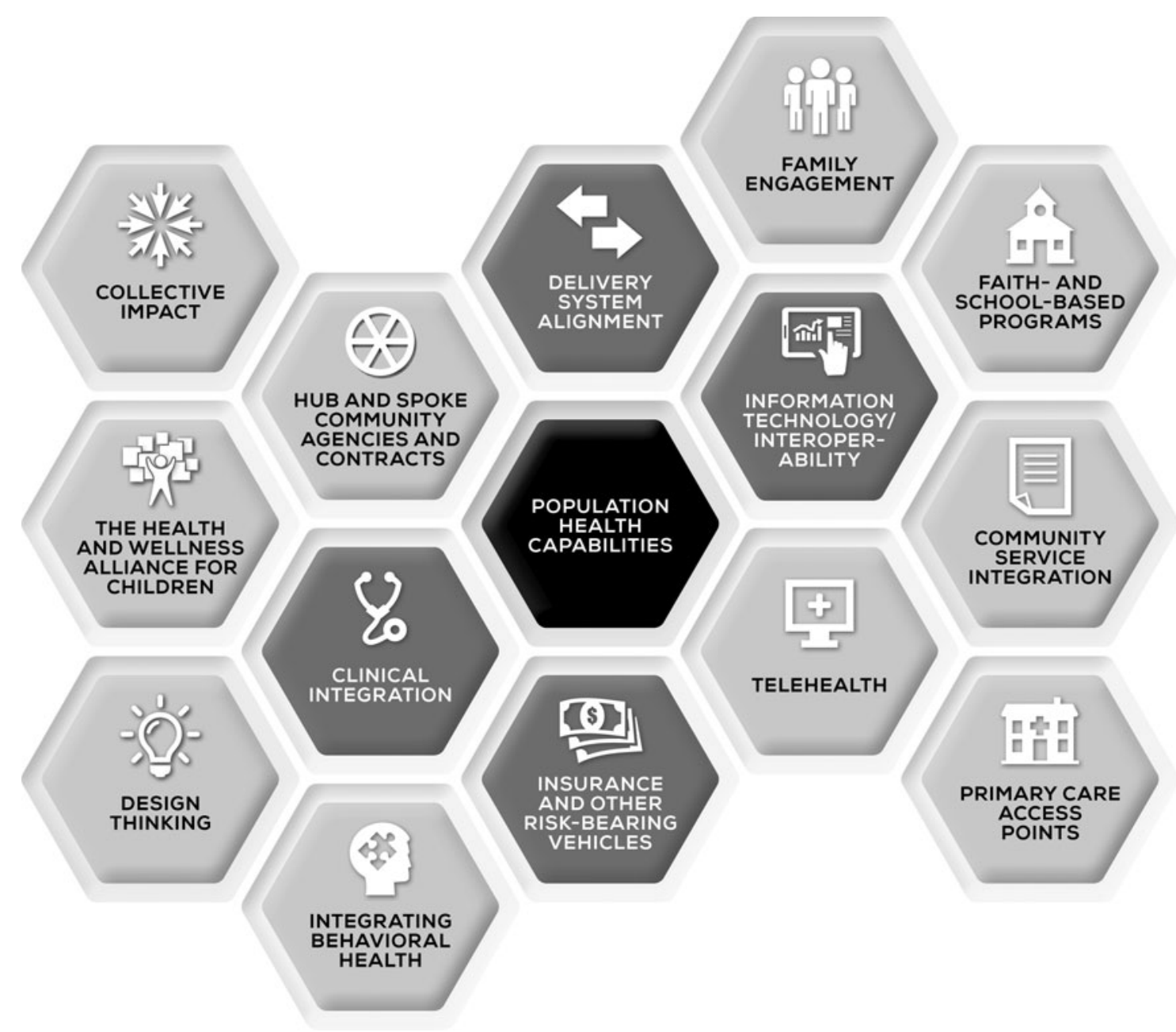

FIG. 1. Population health capabilities. Core; $\square$ Adjacent.

physicians about their own fee-for-service patient populations' care and outcomes compared to benchmarks. In the experience of Children's Health, having the clinically integrated network spend a year contributing and reviewing electronic medical record (EMR) data accelerated the first upside risk performance opportunity through the ACO, with $66 \%$ of network physicians earning incentives the following year.

Although Children's Health is early in its journey, enrolling and assuming risk for covered lives in the provider-owned HMO has galvanized attention on successful population health management with the enrolled population - an outcome that had not occurred under fee-for-service reimbursement systems.

\section{Clinical integration}

The most fundamental capability needed for value-based care, clinical integration includes developing a care delivery support system that will serve patients across an entire continuum of clinical care (Fig. 2). The objective is to facilitate the coordination of patient care across different conditions, providers, settings, and time in order to provide safe, timely, effective, efficient, equitable, and patientfocused care. ${ }^{2}$ This capability requires, at a minimum, the ability to track and communicate patients' clinical and/or claims data across different settings and populations.

Health systems may find they already have the functions needed to assemble a comprehensive clinical integration support system, if the existing functions can be consolidated and repurposed to support a value-based care system. Making the incremental changes necessary to create one source for all care management functions may be challenging, but this change will result in consistent patient/ family communication and better results, while avoiding redundant and confusing outreach.

Small or intermediate-size physician groups may struggle with the incremental expense associated with a robust clinical integration strategy and experience challenges with practice changes. Children's Health offered physicians in its network a low-cost EMR alternative for their practices, implemented an after-hours nurse line, and utilized a central care management function to follow patients across both medical and community settings to achieve greater integration between population health management practice changes and the delivery of care.

The organization built a single clinical integration support system to address the needs of its insurance products as well as the needs of the affiliated delivery system. It repurposed 


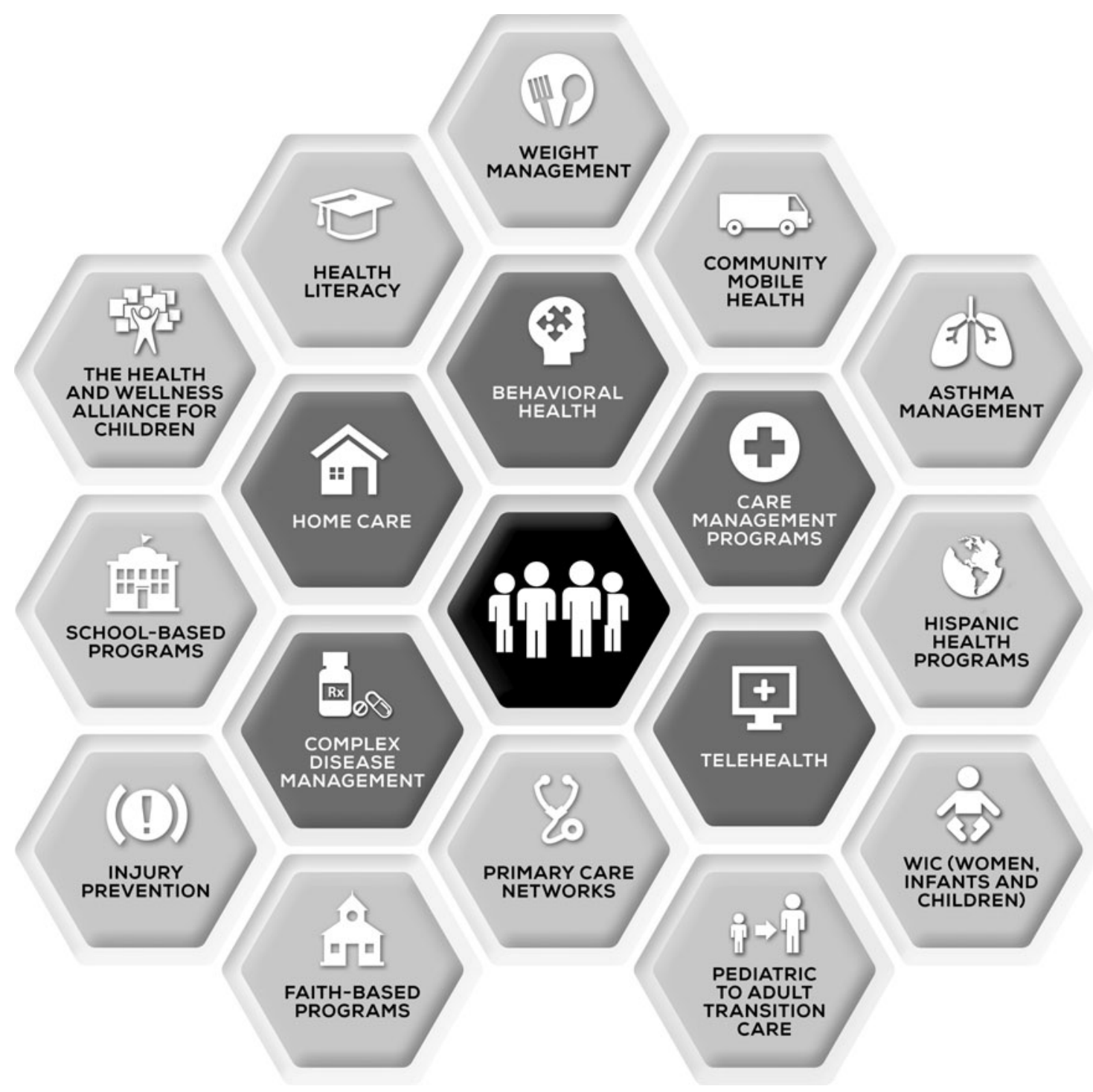

FIG. 2. Clinical integration capabilities. $\square$ Core; $\square$ Adjacent.

and expanded a number of the system elements from existing health system functions, such as health literacy, safety and prevention, patient transitions, care management, and disease management. Intermediate outcome measures for disease management programs in weight management and asthma management are promising as progress toward the future state objectives. The weight management intervention Get Up and Go, involving parents and siblings as well as patients, served 903 referred families in the past 3 years, with $88 \%$ of the participants improving health behaviors, $31 \%$ decreasing their body mass index (BMI) percentile, and $77 \%$ increasing their Progressive Aerobic Cardiovascular Endurance Run test score. The family-based intensive asthma program served more than 650 referred families in the past 2 years and achieved a 90\% reduction in asthmarelated inpatient admissions and an $88 \%$ reduction in emergency room visits for the cohort.

Children's Health also is experimenting with lower-cost options, such as using community service agencies and community health workers instead of nurses, to deliver education and training, using telehealth and mobile technology apps, and inviting families themselves to mentor other families.

A strategy for integrating behavioral health assessments and services into the clinical enterprise is critically important for success in a risk-based environment. Patients who have behavioral issues along with medical illnesses cost more to treat than those with medical conditions only. ${ }^{3}$ The organization found it beneficial to fully integrate behavioral health into each primary care site; however, which model is selected is less important than the urgency of launching a strategy.

Creating flexibility and multiple modalities to connect with patients and families provides more opportunities to identify and address clinical issues sooner and avoid expensive emergency services. Children's Health had a number of primary care practices and school systems test a virtual primary care and behavioral health visit platform (ie, telehealth), which will soon be available across the entire clinically integrated network, allowing physicians to conduct secure, real-time video visits with patients and families. 


\section{Delivery network alignment}

Health care organizations that have invested in population health infrastructure also can leverage their population health assets to help affiliated physicians improve physician performance on risk-based metrics. Health care organizations also may leverage their ability to assume global risk through an ACO on behalf of affiliated providers, then titrate downstream risk assumption by individual providers.

Assembling the provider network for an ACO arrangement usually involves both the opportunity and the challenge of forging strategic relationships with other health providers in the market - key assets that in the future almost all health care providers will be required to have under any circumstance. Decisions include the source of the population health infrastructure, a quality improvement program, pricing methodology, governance structure, and which providers to include and exclude. Children's Health structured an affiliation with an adult provider independent practice association to form a complete health insurance offering. It then developed a series of formal contracts between its financing vehicles and physician groups, to clarify mutual expectations of performance and measurement for its enrolled populations. The ACO governing board is led by elected physician representation from participating network physicians, and detailed committee work for the network is conducted by physician committees.

Initial efforts were focused on aligning clinical objectives and improving performance in the Children's Health Pediatric Group, which serves as the backbone of the primary care medical home delivery system. From 2012 to 2015, Children's Health Pediatric Group was expanded from 6 to 18 clinics. Focusing on reducing the burden of asthma for children in those practices, the organization integrated its population health management capabilities with this delivery system and achieved a $19 \%$ improvement on the Asthma Opportunity Achieved score, a composite of evidence-based asthma practices and a leading indicator of quality care and impact. Although primary care physicians are the "quarterbacks" of care and well-being, specialists control the bulk of the medical spend, so both must be engaged in clinical integration - a reality that creates a higher level challenge that is key to care coordination success with whole populations. This is a future strategy for delivery system alignment.

\section{Information technology}

The organization recognized that the information and technology requirements for a community-based population health strategy needed to enable action from a holistic view of the individual. Therefore, the technology solution needed to include software to stratify insurance claims data, identify high-risk individuals, and enable the workflow of care managers. Further, it would need to eventually integrate and analyze data from multiple EMR systems and collect and share data on the nonmedical determinants of health and community resources. Addressing nonmedical determinants of health places new demands on connectivity, the types of data being stored and transmitted, and on technical solutions. This functionality is not easily obtained in the market and is one Children's Health is weaving together with a variety of systems and interfaces.
A combination of medical and social data about a person and his or her family makes up the optimal picture needed by care managers, health coaches, and navigators. The goal is to provide a single source of information about the individual and family so that both traditional and nontraditional caregivers can implement the best solutions for problems ranging from the simple to the complex. These data exist in many different locations, and for Children's Health, this meant that 3 overlapping building blocks would comprise the information technology structure of the population health information system.

The first is EMR data from providers inside and outside of the Children's Health organization. To enhance understanding of a patient's overall clinical history, clinical information was aggregated by multiple care providers for a given patient in an internally constructed data warehouse. Increasingly, EMRs also can be configured to integrate some socioeconomic information, such as answers to a simple social determinant questionnaire, to enable enhanced understanding by clinical providers. The next step for Children's Health will be to link with social service agencies to make electronic referrals and record service delivery. Children's Health also created a patient navigator role that can be used by the patient and family to link traditional and nontraditional caregivers. This navigator helps to determine gaps in care but also looks at the nonmedical determinants of health for that particular patient and family.

Second is the Interoperable Data Layer, an enhanced data warehouse functionality providing alignment and information integration among the patients/members, various health plans and insurance companies, the community, and the primary care network and other providers. When fully implemented, this platform will facilitate health plan member navigation and traditional provider data needs, house analytics and outcomes measurements, and link community organizations with both providers and consumers. Its goal is to facilitate improved health outcomes, consumer engagement, and reduced operating cost by linking the various stakeholders (sometimes called "uncommon partners") in support of specific families. By linking much of the data in one place, Children's Health's care managers and navigators are able to determine the clinical gaps in care from the EMR data, perform risk stratification and prediction from the claims data software module, and implement complete population health analytics through internally developed data queries.

The third building block is the provider-owned Medicaid health plan information system, which by law must function independently from other provider care management systems. It comprises traditional health plan functions, including claims payment, utilization management, case management, and preventive and wellness care, but does not have functionality for member navigation and communication with community organizations that is provided by the Interoperable Data Layer. Children's Health added an inhome health assessment for its Medicaid HMO enrollees, providing insight into aspects of the family life not obtained from traditional EMR or claims data.

At this time, Children's Health care managers and patient navigators manually integrate data from the different systems to support individual patients and families, as well as to analyze populations more comprehensively. As the Interoperable Data Layer matures and evolves, it will support more efficient bilateral communication. 


\section{Adjacent Capabilities}

The traditional clinical integration support system described earlier primarily addresses the medical determinants of health. It has been known for years, from well-documented literature, that nonmedical determinants (eg, social, behavioral, environmental) have a far greater influence on health, especially in children. The influence of these nonmedical determinants of health makes it virtually impossible for health care systems or health plans alone to sustainably improve the health of a population or community, even in the context of a fully developed medical home network. The challenge of addressing nonmedical determinants of health will continue to exist for health care providers under valuebased arrangements whether the Affordable Care Act stays in place, changes dramatically, or is rescinded altogether.

Yet health care systems have struggled to build models that tightly integrate the medical care world with the social service world - an integration that is necessary to address the whole person or the whole family. ${ }^{4}$ Children's Health found it important to develop 2 of the adjacent capabilities earlier, rather than later, in order to advance its population health strategy more effectively.

\section{Community service organization integration}

The organization developed the Health and Wellness Alliance for Children (the Alliance), a cross-sector coalition of more than 90 organizations serving children and families in Dallas, in late 2012. Since then, this coalition has aligned community organizations around children's health problems, specifically asthma (affecting about 60,000 children in Dallas County), in a Collective Impact ${ }^{5}$ model. The number of children who visited the Children's Health emergency room with a primary diagnosis of asthma was reduced by $49 \%$ over a 4 -year period through the Alliance's systematic, comprehensive effort to link community, public health, philanthropy, education, environmental, social, government, and clinical programs behind a targeted series of interventions (ie, "silver buckshot" instead of a "silver bullet"). These results demonstrate the power of forging uncommon partnerships to improve family health and well-being. ${ }^{6}$

The Alliance also has successfully advanced policy and advocacy to improve the health of children and families. Working with the regional Environmental Protection Agency, Dallas Area Interfaith, the local housing authority, impacted families, and many others, it developed modifications to the city housing code that would improve conditions in rental properties related to mold, pests, and other asthma triggers. The changes were passed by the City Council in 2016 and are currently being implemented. Absent a unified and persistent voice, this public health issue likely would be stymied because of inertia and/or lobbying by affected landlords. The authors have found that a diverse set of organizations working together consider more perspectives and become more effective as advocates.

\section{Engagement of children and families}

Health care systems and insurance companies alike tend to focus on an individual patient or member without regard to the context of the family unit and its powerful influence on health behaviors. This is particularly the case for children, whose behaviors are shaped by their early environment. Children's Health engaged the Business Innovation Factory to apply design thinking principles to develop a deeper understanding of families in the Dallas area. ${ }^{7}$ It worked with more than 200 families - shadowing them at doctors' appointments, the grocery store, church, and in their homes. Groups of families came together to share their challenges and cocreate solutions. The following information was learned during that process:

- Families are primarily focused on a better life, and health needs to be approached in that context.

- Rather than focus on an individual child, the leverage point for sustained behavior change is the family in the context of their community life (eg, school, work, church).

- Families want to break out of their dependence on organizations and be provided with the tools to take charge of improving their own well-being.

These learnings shifted the organization's perspective and helped guide it to develop new capabilities for its population health infrastructure. Children's Health is training and deploying health coaches and family navigators to provide families with tools and a framework - enabling them with a greater level of self-direction in addressing both the medical and nonmedical determinants of their health and well-being.

From that feedback, the organization also evaluated how to be most effective meeting children and families in the context of their community life. It launched a school-based telehealth program in 2013 in underserved areas of Dallas that is reaching over 100 schools and has logged nearly 8000 virtual visits. School nurses are connected via secure, encrypted technology with primary care providers in the Children's Health network. School nurses report that about two thirds of the children seen would otherwise have visited emergency departments or urgent care centers. The program reduces absenteeism at school, keeps parents at work, and improves coordination among home, school, and physicians. The organization also sponsors an interactive and engaging technology platform in north Texas schools that provides for spurts of physical activity in the classroom. Over $42 \%$ of enabled classrooms are active users of the technology, equating to more than 200,000 students. An independent study of use of this technology found improvement in reading scores among users (controlled study); a separate analysis noted improvement in normal BMI among school-age children in districts in the region using the program, attributing that improvement, at least in part, to use of the technology in school. ${ }^{8}$

\section{Lessons Learned}

- In almost every case, the local market will be the driving force for developing strategies in population health-even for those systems with a regional, national, and international presence. A focus on the local market is often at odds with acute care referral and market penetration strategies that continuously seek to expand an institution's reach. However, the local market dynamics need to guide both the scope and scale of population health, as much for "defense" as "offense." Local referral patterns can and will be disrupted eventually by those who are successful at capturing area populations; moreover, as risk-sharing arrangements move further upstream, organizations can expand their reach to individuals who do not require acute care, both in terms of influence on health and per member per month premium revenue. 
- Orienting both governance and executive leadership to the fundamentals of population health is an ongoing process, one made more difficult by the need to live simultaneously in the worlds of fee for service and population health. Regular, routine communication of the population health strategy is necessary, along with the reality that movement in most markets will be gradual. Competing budget and market priorities will most certainly occur during this time, and it will be tempting to approach solutions with an "either-or" rather than a "both-and" attitude. Making realistic long-term financial projections is key to navigating these challenges successfully, as are frank discussions regarding the organization's tolerance for risk and how to optimally manage it.

- Embrace families - the customers - as their own change agents. It is critical to adopt a beginner's mindset with customers; one cannot possibly know what families need without asking them and involving them in the process. In the design studio critiques, families frequently shared that although the solution(s) presented might be interesting, and even elegant, the problem was not identified correctly. Therefore the solutions developed were of limited use to the customers. The organization also routinely underestimated the capabilities of families to act successfully in their own behalf. It needed to change its approach to its customers - to do things with them, not for or to them.

- Creating a culture of health in a community requires servant leadership and collaboration among "uncommon partners." The Children's Health brand is strong and connotes its expertise in medical care and the assurance that it will provide its patients the highest quality medical care. Pivoting to a culture of health in a community means that medical care is only a small portion of what families need to achieve their goals. And the work cannot be done by a single organization, no matter its competence and aspirations. Linking arms with others and putting brand aside in furtherance of the collective community efforts is not "business as usual," but these are essential steps to advance community health.

\section{Summary}

Organizations that take initial steps in building a population health enterprise tailored to the local market, including both infrastructure and risk-based contracts to learn with smaller populations, will be best prepared for the coming industry transition. Whether elements of the infrastructure are repurposed from existing functions or built from scratch, the population health system should serve the entire organization and its goals to maximize its effectiveness. As these elements are defined, the organization can then identify which capabilities are core and critical to its success.

Children's Health found that to understand and begin to address the nonmedical determinants of health, it became important to develop a community integration strategy, even as the core population health infrastructure was being built. Coupled with systems that routinely and effectively engage families in their own health and well-being, the community integration strategy is emerging as a fundamental building block to adopting population health within the organization.
The foundational approach is to connect insurance products, the health care delivery system, and community service agencies around the family. Operating independently, each of these systems is not sufficient to create a tipping point of value for customers, because each addresses only a part of the Triple Aim. Working effectively together on behalf of the family, these systems form a distinct competency that allows Children's Health to deliver true value, effectively steward its resources, and differentiate competitively in the marketplace. Adapting and progressively transforming its business model provides the best foundation for keeping the Children's Health mission promise of making life better for children and their families where they live, work, pray, and play.

\section{Author Disclosure Statement}

Mr. Durovitch and Mr. Roberts declared that there are no conflicts of interest. Funding for this work was provided by Children's Health System of Texas.

\section{References}

1. Business Innovation Factory. An Exploration of a FamilyCentered Business Model. https://urldefense.proofpoint.com/ v2/url?u=https-3A_drive.google.com_open-3Fid-3D0BwWx WgLU2-2DGOS01ldUwzRmN3b1E\&d=CwMFaQ\&c=kqI5qPZzvUeSSdLn1ryw389qklqJeUVcvQsGhZJcVE\&r= LZj4ZIZUIgTEDo6oQnCEsLYXkhzo8gcphknaz45Upnw\&m= FvAVvocD5PSeliKRYao0DdG8dBfOWaPUtbgwHlqUcn0 $\& \mathrm{~s}=2$ WoWAsAMtMWEv9HzOhXhdwGhDu68KnsJWjOplC0c Sgc\&e Accessed March 7, 2017.

2. American Hospital Association. Clinical integration. www .aha.org/advocacy-issues/clininteg/index.shtml. Accessed October 2, 2016.

3. Klein S, Hostetter M. In Focus: integrating behavioral health and primary care. Quality Matters Archive, Aug/Sept 2014. www.commonwealthfund.org/publications/newsletters/qualitymatters/2014/august-september/in-focus Accessed October 2, 2016.

4. Booske B, Athens J, Kindig D, Park H, Remington P. Different perspectives for assigning weights to determinants of health. County Health Rankings Working Paper. Madison, WI: University of Wisconsin Population Health Institute, 2010.

5. Kania J, Kramer M. Collective impact. Stanford Social Innovation Review 2011;1:36-41.

6. Brands C, Roberts P, Shaum R. Attacking childhood asthma: a Dallas success story. www.beckershospitalreview.com/ docs/asthmawhitepaper.pdf Accessed March 7, 2017.

7. Brown T. Design thinking. Harvard Business Review 2008; 6:84-92.

8. Cook Children's. In-classroom physical activity studies show reading scores are up, BMI is down. www.marketwired .com/press-release/in-classroom-physical-activity-studies-showreading-scores-are-up-bmi-is-down-2199072.htm Accessed March 7, 2017.

Address correspondence to: Christopher J. Durovich, MS, MBA Office of the CEO

Children's Health System of Texas 1935 Medical District Drive-ST7.03 Dallas, TX 75235

E-mail: chris.durovich@ childrens.com 